\title{
HIV-Associated TB Syndemic: A Growing Clinical Challenge Worldwide
}

OPEN ACCESS

Edited by:

Annapurna Vyakarnam, King's College London, UK

Reviewed by:

Sarah Rowland-Jones, John Radcliffe Hospital, UK Joern Schmitz, Beth Israel Deaconess Medical Center, USA

${ }^{*}$ Correspondence: Naveen Patil

Naveen.Patil@arkansas.gov

${ }^{\dagger}$ Maria Theresa Montales and Arun Chaudhury have contributed equally to this work.

Specialty section: This article was submitted to HIV and AIDS,

a section of the journal Frontiers in Public Health

Received: 23 July 2015 Accepted: 10 December 2015 Published: 23 December 2015

Citation:

Montales MT, Chaudhury A, Beebe A, Patil S and Patil N (2015) HIV-Associated TB Syndemic: A

Growing Clinical Challenge Worldwide.

Front. Public Health 3:281. doi: 10.3389/fpubh.2015.00281

\author{
Maria Theresa Montales ${ }^{1 \dagger}$, Arun Chaudhury ${ }^{2,3+}$, Alexandria Beebe ${ }^{3}$, Sowmya Patil ${ }^{1}$ and \\ Naveen Patil ${ }^{1,3 *}$
}

${ }^{1}$ University of Arkansas for Medical Sciences (UAMS), Little Rock, AR, USA, ${ }^{2}$ GIM Foundation, Little Rock, AR, USA, ${ }^{3}$ Arkansas Department of Health, Little Rock, AR, USA

The association of tuberculosis (TB) with human immunodeficiency virus (HIV) infection and acquired immune deficiency syndrome over the past several years has become an emerging syndemic. Approximately $10 \%$ of people living with HIV (PLHIV) with latent TB infection will develop active TB disease each year. In this review, we highlight that this phenomenon is not limited to high endemic regions, such as Afro-Asian nations, but globalization/migration is causing increased case detection even in developed nations, such as the United States. Active screening should be performed for TB in PLHIV. A high degree of clinical suspicion for TB is warranted in PLHIV presenting with fever, cough, and unintentional weight loss. HIV-Mycobacterium tuberculosis (MTB) coinfection is often paucibacillary, precluding diagnosis by conventional diagnostics and/or smear microscopy/culture. Improved detection of pulmonary and extrapulmonary TB is now possible by incorporation of the GeneXPERT MTB/RIF assay (Cepheid Inc., Sunnyvale, CA, USA). The World Health Organization recommends instituting immediate therapy for MTB, in conjunction with ongoing or newly introduced anti-retroviral therapy. Vigilance is required to detect drug-induced organ injuries, and early-treatment-induced immune reconstitution inflammatory syndrome. Collaborating MTB and HIV activities in concentrated HIV epidemic settings should become a high public health priority.

Keywords: coinfections, extrapulmonary tuberculosis, HIV, TB, diagnosis, GeneXpert, AFB

\section{INTRODUCTION}

Mycobacterium tuberculosis (MTB) coinfection in patients pre-infected with human immunodeficiency virus (HIV) and/or with full-blown acquired immune deficiency syndrome (AIDS) is an emergent pandemic (1-3). Due to increased recognition of the morbidity and mortality associated with this coinfection, the World Health Organization (WHO) recommends aggressive approaches for MTB screening during primary visits related to HIV screening and treatment (2). While this state of coinfection is a major public health challenge in resource-constrained settings with a high burden of both diseases, such as those in African and Asian nations $(1,4,5)$, it is being increasingly recognized in the settings of developed nations, including the United States (U.S.) (6-9). WHO estimated 1.5 million tuberculosis (TB) deaths in approximately 70\% HIV-negative and 30\% HIVpositive individuals, which makes MTB the second leading cause of death from an infectious disease and the leading cause of death in the setting of AIDS worldwide (10). This review presents the key 
clinical aspects of HIV-MTB coinfection, including the uncommon presentations, diagnostic issues, and management strategies, and emphasizes the continued need for increased vigilance for intensified case finding.

Per WHO guidelines, all clients attending HIV testing centers and people living with HIV (PLHIV) attending anti-retroviral therapy (ART) centers should be clinically screened for TB symptoms at every ambulatory encounter (2). Although the initiation rates of HIV-positive TB patients to ART are improving case fatality continues to be steep compared to HIV-negative TB patients (2). The chief rationale for this difference may include delays in bacteriological detection of HIV-associated TB, enrollment into ART care or immediacy of ART initiation (2).

Different populations pose group-specific challenges in response to detection and treatment. These populations include pediatric patients, antenatal HIV-infected patients, truckers, female sex workers, and men who have sex with men, refugees, and displaced populations (2). Other challenges exist in overcrowded settings such as mines, prisons, homeless shelters, and opioid substitution therapy centers (2). Social issues, including poverty, are warped into the fabric of clinical course of HIV-MTB coinfection (2). In this review, we emphasize early detection by effective implementation of provider-initiated HIV testing and counseling in TB patients as well as intensified TB case finding among PLHIV and initiation of prompt treatment to minimize morbidity and mortality (2).

\section{DEMOGRAPHIC FACTORS RELATED TO MYCOBACTERIUM TUBERCULOSIS COINFECTION IN HIV PATIENTS}

Gender, age, socioeconomic status, marital status, and educational level are factors that impact the likelihood of HIV-MTB coinfection. Male gender is reported to be positively correlated with MTB infection at a ratio of 2:1 (11). HIV-MTB coinfection is more common in adults with an average age of 33-45 years (2). Single status, low socioeconomic and income status, and lack of education are associated with disadvantaged living conditions. Compromised sanitation and poor access to healthcare negatively impact outcomes, which may explain the higher incidence and mortality in this group of patients (2).

It is important to outline some exceptions here. For example, young people may also be afflicted with the coinfection. Pediatric populations may particularly be at high risk, especially those who acquired infections by vertical transmission (12). Interestingly, certain geographic regions such as the Pakistan-Afghanistan border areas report more incidence in females than males (13). According to the WHO 2013 Global TB Report, Afghanistan is a high burden country for TB with a male to female ratio for $\mathrm{TB}$ of 0.5 , so that in contrast to many other countries, Afghan women are more likely to be infected with TB than men. The estimated incidence of HIV-TB coinfections is relatively low (1 in 100,000) in this group and concentrated mostly among injection drug users (according to the World Bank). If there was a higher HIV-MTB coinfection incidence in Afghan women refugees, the observations would likely be due to the higher TB incidence in Afghan women or due to a small sample size of HIV-MTB coinfection in a refugee camp. Furthermore, immunocompromised states, such as those receiving anti-tumor necrosis factor treatment, corticosteroids, dialysis, organ, or hematologic transplantation or those with silicosis, may be more predisposed to HIV-MTB coinfection due to activation of latent TB infection (14).

\section{PATHOGENESIS OF MYCOBACTERIUM TUBERCULOSIS-HIV COINFECTION}

The details of immune responses to TB, HIV, and coinfections have been described in recent reviews $(1,15)$. It is important to note that MTB occurs earlier in HIV patients than other opportunistic infections (OIs) due to increased susceptibility of MTB-specific CD4+ T-cells to HIV infection (16). We present the immune pathogenesis in relation to the intensity of the clinical presentation of TB with pre-existing HIV infection.

Tuberculosis infection is a result of the interplay between bacterial virulence and host resistance $(17,18)$. The infection begins through inhalation of air droplets containing approximately 1-200 bacilli from an individual with active MTB (pulmonary) disease (18). The bacilli are rapidly phagocytosed by resident macrophages in the alveoli. This triggers an inflammatory cascade, followed by development of granuloma. Furthermore, cellmediated immunity through activation of CD4-T lymphocytes is important in the prevention of MTB disease's acceleration and reactivation $(1,15,17,18)$.

On the other hand, HIV transmitted primarily through genital fluids, blood, and mucosa interacts with different cells in the body and tends to escape the host immune response against it, resulting in full-blown AIDS disease (19). Progression of HIV infection is a result of a combination of CD4+ T lymphocytes depletion and a chronic state of immune inactivation. The repression of CD4+ T cells and impairment of macrophages' activity in HIV/AIDS results in down-regulation of the body's immune response to infections, such as MTB. Mycobacteria are contained within granulomas; however, their disruption leads to MTB bacterial growth and systemic dissemination to multiple organs $(1,15)$. MTB has a negative impact on the immune response of the body to HIV by up-regulating the immune response of the host by activating T-cells. Studies have demonstrated that MTB enhances HIV viral replication by increasing the expression of receptors (e.g., CXCR4), which favors viral growth $(15,19)$. The immune response is responsible for the vigor of TB infection in a HIV-coinfected host and is responsible for miliary and extrapulmonary presentations and its associated diagnostic dilemma (1, 15, 20, 21).

Studies aiming to obtain direct evidence of disease progression have been limited due to economic reasons of HIV viral load estimation, especially in countries where the incidence/ prevalence of HIV-MTB coinfection is high but has the shortcoming of resource constraints (22). As pointed out, the impact of MTB on HIV disease progression primarily involves upregulated 
HIV-1 viral load, including the development of new OIs (22-28). Interestingly, TB was found to exert significant effect on diminishing survival rates in subjects with more preserved immunological status (i.e., CD4 cell counts $>200$ cells $/ \mu L)(27,28)$. Enhanced HIV-1 production has been demonstrated at local sites of MTB infection, for example, in bronchoalveolar lavage (BAL) fluid from TB involved, compared with uninvolved, lungs of patients with HIV-1/TB coinfection (29). A clinical presentation of TB that is particularly observed in HIV-1-infected patients is pleural TB, a common presentation in African coinfected patients $(30,31)$. These sites of active MTB infection act as foci of HIV replication and evolution of quasi-species, independent of systemic HIV-1 activity, and may be responsible for disseminated MTB infection in HIV-1 coinfected hosts.

Mycobacterium tuberculosis breaches the alveolar epithelium during the first phase of extrapulmonary dissemination. Molecular mechanisms for this cytolysis have been reported. For example, heparin-binding hemagglutinin adhesin (HBHA) facilitates MTB to bind to sulfated glycoconjugates on epithelial cells. Two gene products of the MTB RD1 gene, early secretory antigenic target $6 \mathrm{kDa}$ (ESAT-6) and culture filtrate protein $10 \mathrm{kDa}$ (CFP-10), have been causally linked to the cell lysis and extrapulmonary mycobacterial spread (32). The trafficking of mycobacteria to the regional lymph nodes, while essential for the development of a protective T-cell-mediated immune response, is the first extrapulmonary site of migration of MTB. Bacteria thereafter disseminated through the bloodstream and lymphatics lead to extrapulmonary tuberculosis (EPTB). Lung granulomas from MTB/HIV-1 coinfected patients release lower levels of in situ TNF production (33). Additionally, MTB-HIV-1 coinfected hosts have low circulating mannose-binding lectin levels (34). The complex interactions that take place between host $\mathrm{T}$ cells, Tregs, cytokine production, and overall impaired Th1 responses predispose to extrapulmonary infections in HIV-coinfected hosts $(35,36)$. Several primary immunodeficiencies, including Mendelian susceptibility to mycobacterial infections, enhance the overall risk of EPTB (37). These aspects merit detailed studies in the future.

\section{SCREENING FOR MYCOBACTERIUM TUBERCULOSIS-HIV COINFECTION}

World Health Organization formulated a policy on collaborative MTB/HIV activities with the chief aim to reduce the dual burden of MTB and HIV (2). WHO recommends MTB screening among HIV-positive patients at the time of diagnosis and before the initiation of ART. The screening for MTB should also be extended to the household contacts of HIV-positive patients (2). The screening tool utilizes a clinical symptombased algorithm that consists of the absence or presence of current cough, fever, weight loss, or night sweats at the time of initial presentation and at every visit to health clinics. This tool can be used to identify patients who need further medical attention and those who need MTB chemoprophylaxis intended to prevent active TB disease (rather than prevention of TB infection per se).

\section{DIAGNOSIS OF MYCOBACTERIUM TUBERCULOSIS INFECTION IN HIV- COINFECTED PATIENTS}

A high degree of clinical suspicion and the need for obtaining a focused and detailed history merits emphasis. Numerous diseases may mimic presentations of TB, but TB should be high up on the differential for pneumonic presentations and HIV-associated thoracic diseases $(7,38)$. Initial screening with a tuberculin skin test (TST) or interferon-gamma release assays (IGRA) is recommended. TST, which can be affected by bacillus Calmette-Guérin (BCG) vaccination, and/or IGRA, which is unaffected by BCG vaccination, are routinely used screening methods for TB in asymptomatic, non-HIV-infected individuals in low TB prevalence nations. However, it may be noted that these tests have a "low specificity" (TST) or "have false positive and false negative results" (IGRA) and are of low diagnostic values in TB endemic areas, especially when there is coinfection with HIV. When there is a high degree of clinical suspicion or pneumatic presentations, the standard diagnosis of TB in symptomatic patients is typically not a TST or IGRA, but rather achieved with sputum smear microscopy. This is inexpensive, rapid, and easy to perform in a field setting but has a lower sensitivity in MTB-HIV coinfection. The sputum smear sample has the advantage of being used for culture of MTB and drug susceptibility testing. Because coinfection lowers microscopy sensitivity, several current diagnostic techniques are more sensitive than the conventional sputum smear microscopy in detecting MTB in HIV-positive individuals. A growth-based detection of MTB, in cultures and molecular techniques, such as nucleic acid amplification testing (NAAT), has been shown to be more sensitive and allows strain characterization and drug susceptibility tests $(39,40)$.

In 2013, the Food and Drug Administration (FDA) in the U.S. approved the GeneXpert MTB/RIF assay (Cepheid Inc., Sunnyvale, CA, USA), a NAAT-based diagnostic platform. WHO endorsed the use of this assay as the initial test for TB diagnosis in PLHIV or who are suspected of multidrugresistant TB (MDR-TB) and has since been extensively used (41-44). The cost of the test, despite being subsidized, has been a barrier in many TB endemic countries to be used as a first line diagnostic tool.

Additionally, low thresholds should be exercised when performing standard chest $\mathrm{X}$ rays and computerized tomography scans for diagnostic suspicion of miliary TB and detecting lesions during extrapulmonary manifestations (38). The pleomorphic manifestations of radiographic appearances in HIV-MTB coinfection is depicted in Figure 1.

A critical caveat in the diagnosis of MTB relates to the context of latent TB. The low specificity of TST and the issues of false positive and false negative detection with IGRA's [QuantiIFERON ${ }^{\circledR}$-TB Gold In-Tube (QFT-G-IT) assay (Cellestis Ltd., Carnegie, VIC, Australia) or T-SPOT ${ }^{\circledR} T B$ assay (Oxford Immunotec Inc., Marlborough, MA, USA)] pose significant challenges in HIV-coinfected patients. The critical aspects and unmet needs in diagnosing latent TB have been recently reviewed (39). Due to very high risk of activation of latent TB in HIV coinfection, 

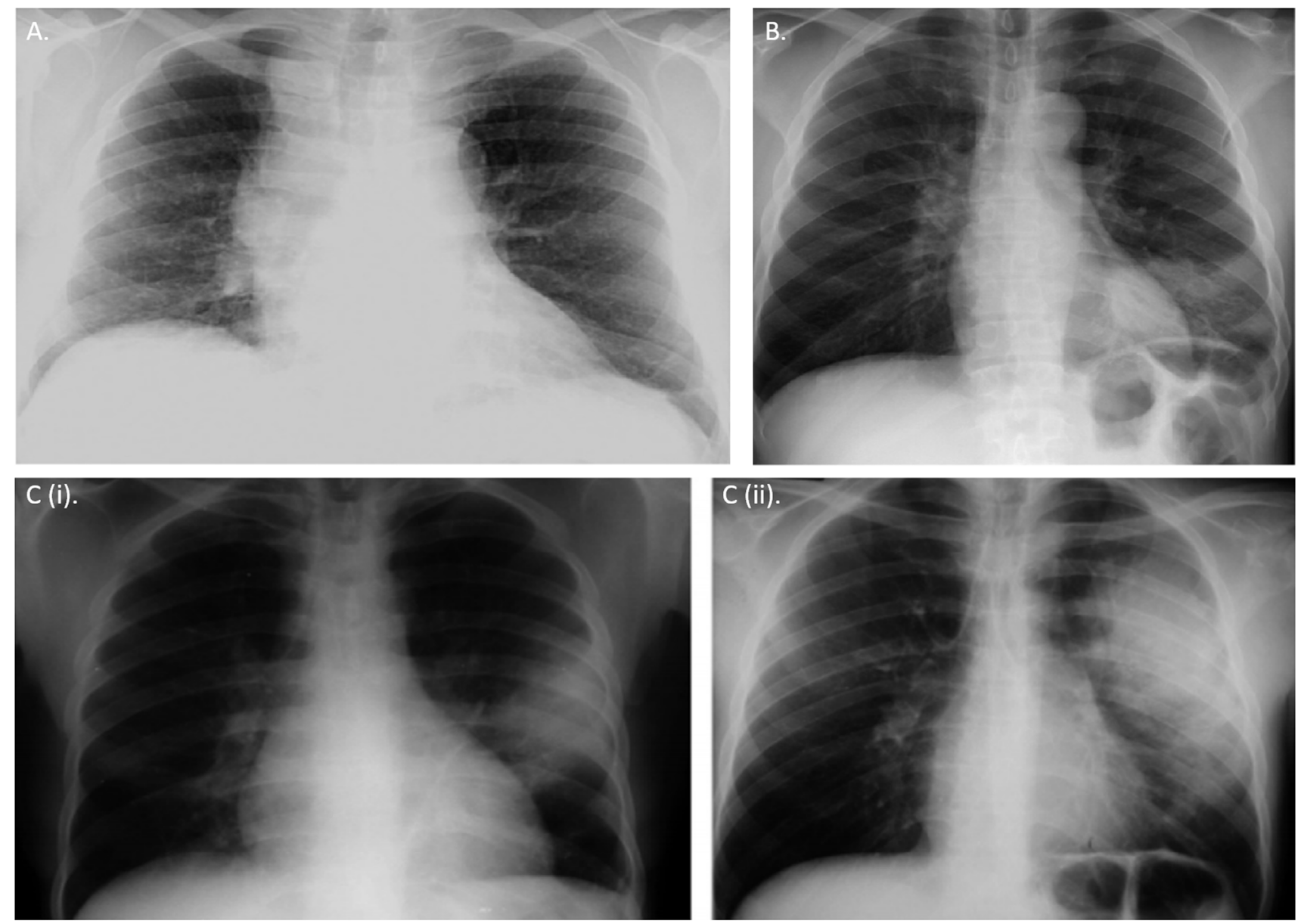

FIGURE 1 | Chest radiographic appearances of tuberculosis in HIV. (A) Right hilar lymphadenopathy and bronchial markings in a primary pulmonary tuberculosis-like presentation in a HIV-infected young adult (B) Left lower lobe consolidation due to Streptococcus pneumonia infection in pre-existing tuberculosis and HIV coinfections $\mathbf{( C i , i i ) ~ R a d i o l o g i c ~ a p p e a r a n c e ~ o f ~ I R I S . ~ C h e s t ~ X - r a y ~ s h o w i n g ~ r a d i o g r a p h i c ~ p r o g r e s s i o n ~ o f ~ a ~ s m a l l ~ l e f t ~ l o b a r ~ l e s i o n ~ t o ~ w i d e s p r e a d ~ c o n s o l i d a t i o n ~ o f ~}$ a tuberculosis patient few weeks after initiating anti-retroviral therapy (ART). All CXRs obtained from permission with Chou et al. (38).

these cohorts (immunocompromised subjects, immigrants from countries with high disease burden, homeless subjects, prisoners, illicit drug users, healthcare workers taking care of high risk group populations, and adult contacts of persons with TB) need careful identification and evaluation to be considered for followup, preventive therapy, and prompt institution of treatment upon detection of active lesions.

\section{CLINICAL MANAGEMENT OF MYCOBACTERIUM TUBERCULOSIS-HIV COINFECTION}

Delay in the diagnosis of MTB in HIV-positive patients and inadequate initial treatment may lead to multidrug resistant $\mathrm{TB}$ (MDR-TB). MDR-TB is a growing concern not only because of its longer treatment duration but also because of its associated increased transmission risk among contacts and increased mortality rates in HIV-coinfected patients (45).
Considerations in the management of HIV-MTB coinfection include adjustment in the duration, dosage, and frequency of anti-TB drug administration as well as optimal timing of initiation of highly active anti-retroviral therapy (HAART) treatment. The standard therapeutic regimen for TB consists of isoniazid (INH), a rifamycin (rifampin-RIF or rifabutin-RFB), pyrazinamide (PZA), and ethambutol (EMB) given for 2 months, followed by INH plus rifamycin for 4-7 months (46). Treatment of active $\mathrm{TB}$ infection is the priority due to the risk of transmitting TB to other people. However, when CD4+ T cell count is extremely low $\left(<50\right.$ cells $\left./ \mathrm{mm}^{3}\right)$, an appropriate treatment plan should be formulated wherein prompt initiation of HAART is necessary. Randomized controlled trials such as SAPiT (47), CAMELIA (48), and STRIDE (49) suggested and have provided evidence for immediate institution of ART within 2 weeks of starting antimycobacterial therapy.

There are several debates on the ideal timing of HAART initiation and concomitant administration with anti-TB medications. Advantages of early HAART administration include higher cure 
rates, reduced risk of relapse, reduced risk of infection with other $\mathrm{HIV}$-associated OIs, and lower mortality rates. On the other hand, disadvantages include potential drug interactions of HAART with rifampicin, thus limiting co-administration of selected protease inhibitors (PIs), cumulative toxicity, therapeutic failure, and the risk of immune reconstitution inflammatory syndrome (IRIS), which affect the long-term adherence to HAART in MTB-infected patients (50). Furthermore, non-compliance due to pill burden, side effects of medications, accessibility of treatment centers for HIV and TB, fears of stigmatization, cost of healthcare, and lack of proper health education are also major problems that need to be addressed to successfully treat MTB patients (51). In general, the priority should be to provide directly observed therapy (DOT) for these patients to ensure compliance and treatment success (10). Two recently published trials have re-emphasized the importance of early initiation of ART. The TEMPRANO study from the Ivory Coast showed that early ART initiation in subjects with CD4+ cell count of $\geq 500$ cells $/ \mathrm{mm}^{3}$ was associated with a $44 \%$ lower risk of death or severe HIV-related illness than when ART was initiated according to prevailing WHO criteria (52). Furthermore, patients who received INH prophylaxis had a 35\% lower risk of death or severe HIV-related illness than patients who did not receive it. In the START study involving 215 sites in 35 countries, the risk of death, a serious AIDS-related event, or a serious non-AIDSrelated event was 57\% less among the subjects treated early than among those treated when the CD4+ cell count decreased to 350 cells $/ \mathrm{mm}^{3}$ (53). In both trials, a reduced rate of TB after early rather than deferred ART was the most significant contributor to the overall benefits. Early initiation of ART possesses the added benefits of reducing the risk of sexual transmission of HIV and HSV2 infections (54). However, the public health challenges concerning the cost and global health motivation for implementation of these recent evidence-based guidelines remains a major challenge that needs to be overcome (54).

In specific patients, such as $\mathrm{HIV}$-infected pregnant women with active TB, the recommendation is to start on ART as early as feasible, both for maternal health and to prevent perinatal transmission of HIV (55). The choice of ART should be based on efficacy and safety in pregnancy and take into account potential drug interactions between anti-retrovirals and rifamycin (50). In HIV-infected patients with latent tuberculosis infection (LTBI), isoniazid preventive therapy (IPT) has been reported to reduce reactivation of latent TB infection in the context of both industrialized and developing countries (56).

\section{COMPLICATIONS ARISING OUT OF THERAPY FOR HIV-MTB COINFECTION}

\section{Immune Reconstitution Inflammatory Syndrome}

Transient exacerbation of respiratory signs and symptoms despite reduction in viral load and/or radiological deterioration may develop in HIV-MTB coinfected patients who are treated with anti-TB medications concomitantly with HAART (19). IRIS has a dimorphic presentation: unmasking and paradoxical $(57,58)$. Restoration of immune competence by administration of ART results in hyperimmune host response to TB bacilli and/or antigens. Unmasking IRIS presents with active TB soon after ART is started. Paradoxical IRIS refers to the worsening of TB symptoms after ART is initiated in patients who are receiving TB treatment. Anti-inflammatory drugs and steroids are the mainstay therapy for IRIS. Discontinuation of HAART is not warranted in most cases. Delaying initiation of ART for 2-8 weeks may reduce the incidence and severity of IRIS. However, this possible advantage of delayed ART must be weighed against the potential benefit of earlier ART in improving immune function and preventing progression of HIV disease and mortality. Importantly, immune reconstitution with ART may result in unmasking LTBI (i.e., conversion of a previously negative TST to a positive TST or a positive IGRA for MTB-specific proteins) (44). A positive IGRA, similar to a positive TST, is indicative of LTBI in the absence of evidence of active TB disease. Because treatment for LTBI is indicated in the absence of evidence of active TB disease, this situation should be clinically recognized, especially in high risk groups.

\section{Anti-Tuberculosis and Anti-Retroviral Drug Interaction}

Rifamycins are potent inducers of the hepatic cytochrome $\mathrm{P}$ (CYP) 450 enzyme. They are associated with significant interactions with all PIs, some non-nucleoside reverse transcriptase inhibitors (NNRTIs), maraviroc (MVC), and raltegravir (RAL) (50, 58-61), leading to enhanced drug clearance and significant lowering in circulating anti-retroviral drugs. However, good virological, immunological, and clinical outcomes may be achieved with standard doses of efavirenz (EFV) and nevirapine (NVP) when combined with rifampin (61). Suboptimal HIV suppression or suboptimal response to TB treatment should prompt immediate assessment of drug adherence, sub-therapeutic drug levels (with consideration for therapeutic drug monitoring), and acquired drug resistance.

\section{Other Known Side Effects}

Hepatotoxicity potentially arises from co-administration of antiretroviral and anti-mycobacterial agents; therefore, continuous monitoring of liver function should be exercised. Symptoms of abdominal pain, jaundice, loss of appetite, fever, and nausea merit urgent clinical attention (39). Additionally, many of these patients may need additional treatment, for example, for drug dependence or HCV coinfection, which presents additional risk for coexisting liver diseases (62). Peripheral neuropathy, on the other hand, can occur with administration of INH, didanosine (ddI), or stavudine (d4T) or may be a manifestation of the native HIV infection per se. All patients receiving INH should be administered supplemental pyridoxine to reduce peripheral neuropathy (61). Other coexisting medical and behavioral conditions, such as tobacco smoking, alcoholism, malnutrition, and diabetes mellitus, may significantly impact disease management and outcomes (63).

\section{CONCLUSION}

Coinfection with the HIV is an important contributing factor to TB mortality not only in many countries, mainly those of 
sub-Saharan Africa, but also in developed countries such as the U.S. Today, caring for TB patients and controlling the spread of TB are complicated by the emergence of MDR-TB. Globalization and migration from endemic zones continues to be a major force in global spread of this coinfection.

\section{REFERENCES}

1. Pawlowski A, Jansson M, Sköld M, Rottenberg ME, Källenius G. Tuberculosis and HIV co-infection. PLoS Pathog (2012) 8(2):e1002464. doi:10.1371/ journal.ppat.1002464

2. World Health Organization. Global Tuberculosis Report. Geneva: World Health Organization (2013).

3. Montales MT, Beebe A, Chaudhury A, Patil N. Mycobacterium tuberculosis infection in a HIV-positive patient. Respir Med Case Rep (2015) 16:160-2. doi:10.1016/j.rmcr.2015.10.006

4. Efsen AM, Schultze A, Post F, Panteleev A, Furrer H, Miller R, et al. Major challenges in clinical management of TB/HIV co-infected patients in Eastern Europe compared with Western Europe and Latin America. J Int AIDS Soc (2014) 17(4 Suppl 3):19505. doi:10.7448/IAS.17.4.19505

5. Sester M, van Leth F, Bruchfeld J, Bumbacea D, Cirillo DM, Dilektasli AG, et al. TBNET. Risk assessment of tuberculosis in immunocompromised patients. A TBNET study. Am J Respir Crit Care Med (2014) 190(10):1168-76. doi:10.1164/rccm.201405-0967OC

6. Hotez PJ. Blue marble health and "the big three diseases": HIV/AIDS, tuberculosis, and malaria. Microbes Infect (2015) 17(8):539-41. doi:10.1016/j. micinf.2015.05.004

7. Khabbaz RF, Moseley RR, Steiner RJ, Levitt AM, Bell BP. Challenges of infectious diseases in the USA. Lancet (2014) 384(9937):53-63. doi:10.1016/ S0140-6736(14)60890-4

8. Patil N, Marco A, Saba H, Samant R, Mukasa L. TB or not TB; don't miss the obvious. J Ark Med Soc (2014) 111(6):112-4.

9. Berzkalns A, Bates J, Ye W, Mukasa L, France AM, Patil N, et al. The road to tuberculosis (Mycobacterium tuberculosis) elimination in Arkansas; a re-examination of risk groups. PLoS One (2014) 9(3):e90664. doi:10.1371/ journal.pone.0090664

10. Corbett EL, Watt CJ, Walker N, Maher D, Williams BG, Raviglione MC, et al. The growing burden of tuberculosis: global trends and interactions with the HIV epidemic. Arch Intern Med (2003) 163:1009-21. doi:10.1001/ archinte.163.9.1009

11. Holmes C, Hausler H, Nunn P. A review of sex differences in the epidemiology of tuberculosis. Int J Tuberc Lung Dis (1998) 2(2):96-104.

12. Venturini E, Turkova A, Chiappini E, Galli L, de Martino M, Thorne C. Tuberculosis and HIV co-infection in children. BMC Infect Dis (2014) 14(Suppl 1):S5. doi:10.1186/1471-2334-14-S1-S5

13. Available from: http://www.who.int/tb/publications/tb_women_factsheet_251013.pdf, 2014.

14. Richeldi L, Losi M, D’Amico R, Luppi M, Ferrari A, Mussini C, et al. Performance of tests for latent tuberculosis in different groups of immunocompromised patients. Chest (2009) 136(1):198-204. doi:10.1378/ chest.08-2575

15. Shankar EM, Vignesh R, Ellegård R, Barathan M, Chong YK, Bador $\mathrm{MK}$, et al. HIV-Mycobacterium tuberculosis co-infection: a 'danger-couple model' of disease pathogenesis. Pathog Dis (2014) 70(2):110-8. doi:10.1111/2049-632X.12108

16. Geldmacher C, Ngwenyama N, Schuetz A, Petrovas C, Reither K, Heeregrave EJ, et al. Preferential infection and depletion of Mycobacterium tuberculosis-specific CD4 T cells after HIV-1infection. J Exp Med (2010) 207(13):2869-81. doi:10.1084/jem.20100090

17. Scaling Up of Collaborative TB/HIV Activities in Concentrated HIV Epidemic Settings. A Case Study from India. World Health Organization (2015). Available from: http://www.who.int/tb/publications/ india_case_study_document_page/en/

18. Cooper A. Cell mediated immune responses in tuberculosis. Annu Rev Immunol (2009) 27:393-422. doi:10.1146/annurev.immunol.021908.132703
HIV-infected patients with active TB disease should receive treatment support, including adherence counseling and DOT, corresponding to their needs. In conclusion, MTB and HIV coinfection remains a diagnostic and therapeutic challenge worldwide.

19. Saharia KK, Koup RA. T cell susceptibility to HIV influences outcome of opportunistic infections. Cell (2013) 155(3):505-14. doi:10.1016/j. cell.2013.09.045

20. Moir S, Chun T, Fauci A. Pathogenic mechanisms of HIV disease. Annu Rev Pathol (2011) 6:223-48. doi:10.1146/annurev-pathol-011110-130254

21. Diedrich C, Flynn J. HIV-1/Mycobacterium tuberculosis co-infection immunology: how does HIV-1 exacerbate tuberculosis? Infect Immun (2011) 79(4):1407-17. doi:10.1128/IAI.01126-10

22. Djoba Siawaya JF, Ruhwald M, Eugen-Olsen J, Walzl G. Correlates for disease progression and prognosis during concurrent HIV/TB infection. Int J Infect Dis (2007) 11(4):289-99. doi:10.1016/j.ijid.2007.02.001

23. Wolday D, Hailu B, Girma M, Hailu E, Sanders E, Fontanet AL. Low CD4+ T-cell count and high HIV viral load precede the development of tuberculosis disease in a cohort of HIV-positive Ethiopians. Ethiop Med J (2003) 41(Suppl 1):67-73.

24. Kaplan JE, Hanson DL, Jones JL, Dworkin MS; Adult and Adolescent Spectrum of HIV Disease Project Investigators. Viral load as an independent risk factor for opportunistic infections in HIV-infected adults and adolescents. AIDS (2001) 15(14):1831-6. doi:10.1097/00002030-200109280-00012

25. SwindellsS,EvansS,Zackin R, GoldmanM,HaubrichR,FillerSG, etal.Predictive value of HIV-1 viral load on risk for opportunistic infection. J Acquir Immune Defic Syndr (2002) 30(2):154-8. doi:10.1097/00042560-200206010-00003

26. Toossi Z. Virological and immunological impact of tuberculosis on human immunodeficiency virus type 1 disease. J Infect Dis (2003) 188(8):1146-55. doi:10.1086/378676

27. Whalen C, Horsburgh CR, Hom D, Lahart C, Simberkoff M, Ellner J. Accelerated course of human immunodeficiency virus infection after tuberculosis. Am J Respir Crit Care Med (1995) 151(1):129-35. doi:10.1164/ ajrccm.151.1.7812542

28. Goletti D, Weissman D, Jackson RW, Graham NM, Vlahov D, Klein RS, et al. Effect of Mycobacterium tuberculosis on HIV replication. Role of immune activation. J Immunol (1996) 157(3):1271-8.

29. Nakata K, Rom WN, Honda Y, Condos R, Kanegasaki S, Cao Y, et al. Mycobacterium tuberculosis enhances human immunodeficiency virus-1 replication in the lung. Am J Respir Crit Care Med (1997) 155(3):996-1003. doi:10.1164/ajrccm.155.3.9117038

30. Toossi Z, Johnson JL, Kanost RA, Wu M, Luzze H, Peters P, et al. Increased replication of HIV1 at sites of Mycobacterium tuberculosis infection: potential mechanisms of viral activation. J Acquir Immune Defic Syndr (2001) 28(1):1-8. doi:10.1097/00042560-200109010-00001

31. Frye MD, Pozsik CJ, Sahn SA. Tuberculous pleurisy is more common in AIDS than in non-AIDS patients with tuberculosis. Chest (1997) 112(2):393-7. doi:10.1378/chest.112.2.393

32. Krishnan N, Robertson BD, Thwaites G. The mechanisms and consequences of the extra-pulmonary dissemination of Mycobacterium tuberculosis. Tuberculosis (Edinb) (2010) 90(6):361-6. doi:10.1016/j.tube.2010.08.005

33. de Noronha AL, Báfica A, Nogueira L, Barral A, Barral-Netto M. Lung granulomas from Mycobacterium tuberculosis/HIV-1 co-infected patients display decreased in situ TNF production. Pathol Res Pract (2008) 204:155. doi:10.1016/j.prp.2007.10.008

34. Garred P, Richter C, Andersen AB, Madsen HO, Mtoni I, Svejgaard A, et al. Mannan-binding lectin in the sub-Saharan HIV and tuberculosis epidemics. Scand J Immunol (1997) 46(2):204-8. doi:10.1046/j.1365-3083.1997.d01-111.x

35. Zhang M, Gong J, Iyer DV, Jones BE, Modlin RL, Barnes PF. T cell cytokine responses in persons with tuberculosis and human immunodeficiency virus infection. J Clin Invest (1994) 94:2435-42. doi:10.1172/JCI117611

36. Berrington WR, Hawn TR. Mycobacterium tuberculosis, macrophages, and the innate immune response: does common variation matter? Immunol Rev (2007) 219:167-86. doi:10.1111/j.1600-065X.2007.00545.x 
37. Casanova JL, Abel L. Genetic dissection of immunity to mycobacteria: the human model. Annu Rev Immunol (2002) 20:581-620. doi:10.1146/annurev. immunol.20.081501.125851

38. Chou SH, Prabhu SJ, Crothers K, Stern EJ, Godwin JD, Pipavath SN. Thoracic diseases associated with HIV infection in the era of antiretroviral therapy: clinical and imaging findings. Radiographics (2014) 34(4):895-911. doi: $10.1148 /$ rg.344130115

39. Getahun H, Matteelli A, Chaisson RE, Raviglione M. Latent Mycobacterium tuberculosis infection. N Engl J Med (2015) 372(22):2127-35. doi:10.1056/ NEJMra1405427

40. World Health Organization. Improving the Diagnosis and Treatment of SmearNegative Pulmonary and Extrapulmonary Tuberculosis Among Adults and Adolescents: Recommendations for HIV-Prevalent and Resource-Constrained Settings (2006). Available from: http://www.who.int/tb/publications/2006/ tbhiv_recommendations.pdf

41. Kaul K. Molecular detection of Mycobacterium tuberculosis: impact on patient care. Clin Chem (2001) 47(8):1553-8.

42. Patil N, Saba H, Marco A, Samant R, Mukasa L. Initial experience with GeneXpertMTB/RIF assay in the Arkansas tuberculosis control program. Aust Med J (2014) 7(5):203-7. doi:10.4066/AMJ.2014.1905

43. Patil N, Montales MT, Mittadodla P, Mukasa LN, Bhaskar N, Bates JH, et al. Tuberculosis reinfection in a pregnant cystic fibrosis patient. Respir Med Case Rep (2015) 16:57-9. doi:10.1016/j.rmcr.2015.04.002

44. Patil N, Marco A, Montales MT, Bhaskar N, Mittadodla P, Mukasa LN. Pulmonary tuberculosis in a patient with cystic fibrosis. North Am J Med Sci (2015) 7:233-5. doi:10.4103/1947-2714.157494

45. Dean AS, Zignol M, Falzon D, Getahun H, Floyd K. HIV and multidrug-resistant tuberculosis: overlapping epidemics. Eur Respir J (2014) 44(1):251-4. doi:10.1183/09031936.00205413

46. Curran A, Falcó V, Pahissa A, Ribera E. Management of tuberculosis in HIVinfected patients. AIDS Rev (2012) 14(4):231-46.

47. Naidoo A, Naidoo K, Yende-Zuma N, Gengiah TN, Padayatchi N, Gray AL, et al. Changes to antiretroviral drug regimens during integrated TB-HIV treatment: results of the SAPiTtrial. Antivir Ther (2014) 19(2):161-9. doi:10.3851/ IMP2701

48. Borand L, Pheng P, Saman M, Leng C, Chea P, Sarady Ay S, et al. [Tuberculosis and HIV co-infection: clinical trial under the coordination of the Institut Pasteur in Cambodia]. Med Sci (Paris) (2013) 29(10):908-11. doi:10.1051/ medsci/20132910020

49. Crump JA, Wu X, Kendall MA, Ive PD, Kumwenda JJ, Grinsztejn B, et al. Predictors and outcomes of Mycobacterium tuberculosis bacteremia among patients with HIV and tuberculosis co-infection enrolled in the ACTG A5221 STRIDE study. BMC Infect Dis (2015) 15(1):12. doi:10.1186/ s12879-014-0735-5

50. Havlir DV, Currier JS. CROI 2015: complications of HIV infection and antiretroviral therapy. Top Antivir Med (2015) 23(1):56-65.

51. Beebe A, Seaworth B, Patil N. Rifampicin-induced nephrotoxicity in a tuberculosis patient. J Clin Tuberc Other Mycobact Dis (2015) 1:13-5. doi:10.1016/j. jctube.2015.09.001

52. TEMPRANO ANRS 12136 Study Group; Danel C, Moh R, Gabillard D, Badje A, Le Carrou J, et al. A trial of early antiretrovirals and isoniazid preventive therapy in Africa. N Engl J Med (2015) 373(9):808-22. doi:10.1056/ NEJMoa1507198

53. INSIGHT START Study Group ; Lundgren JD, Babiker AG, Gordin F, Emery S, Grund B, et al. Initiation of antiretroviral therapy in early asymptomatic HIV infection. N Engl J Med (2015) 373(9):795-807. doi:10.1056/ NEJMoa1506816

54. Abdool Karim SS. Overcoming impediments to global implementation of early antiretroviral therapy. N Engl J Med (2015) 373(9):875-6. doi:10.1056/ NEJMe1508527

55. van Lettow M, Bedell R, Mayuni I, Mateyu G, Landes M, Chan AK, et al. Towards elimination of mother-to-child transmission of HIV: performance of different models of care for initiating lifelong antiretroviral therapy for pregnant women in Malawi (Option B+). J Int AIDS Soc (2014) 17:18994. doi:10.7448/IAS.17.1.18994

56. Ayele HT, Mourik MS, Debray TP, Bonten MJ. Isoniazid prophylactic therapy for the prevention of tuberculosis in HIV infected adults: a systematic review and meta-analysis of randomized trials. PLoS One (2015) 10(11):e0142290. doi:10.1371/journal.pone. 0142290

57. Barber DL, Andrade BB, Sereti I, Sher A. Immune reconstitution inflammatory syndrome: the trouble with immunity when you had none. Nat Rev Microbiol (2012) 10(2):150-6. doi:10.1038/nrmicro2712

58. El-Sadr WM, Tsiouris SJ. HIV-associated tuberculosis: diagnostic and treatment challenges. Semin Respir Crit Care Med (2008) 29(5):525-31. doi:10.10 55/s-0028-1085703

59. Regazzi M, Carvalho AC, Villani P, Matteelli A. Treatment optimization in patients co-infected with HIV and Mycobacterium tuberculosis infections: focus on drug-drug interactions with rifamycins. Clin Pharmacokinet (2014) 53(6):489-507. doi:10.1007/s40262-014-0144-3

60. Abdool Karim SS, Naidoo K, Grobler A, Padayatchi N, Baxter C, Gray AL, et al. Integration of antiretroviral therapy with tuberculosis treatment. $N$ Engl $J$ Med (2011) 365(16):1492-501. doi:10.1056/NEJMoa1014181

61. Available from: https://aidsinfo.nih.gov/guidelines/html/1/adult-and-adolescent-arv-guidelines/32/drug-interactions, 2013.

62. Getahun H, Baddeley A, Raviglione M. Managing tuberculosis in people who use and inject illicit drugs. Bull World Health Organ (2013) 91(2):154-6. doi:10.2471/BLT.13.117267

63. Bates M, Marais BJ, Zumla A. Tuberculosis comorbidity with communicable and noncommunicable diseases. Cold Spring Harb Perspect Med (2015) 5(11):a017889. doi:10.1101/cshperspect.a017889

Conflict of Interest Statement: The authors declare that the research was conducted in the absence of any commercial or financial relationships that could be construed as a potential conflict of interest.

Copyright (๑) 2015 Montales, Chaudhury, Beebe, Patil and Patil. This is an open-access article distributed under the terms of the Creative Commons Attribution License (CC BY). The use, distribution or reproduction in other forums is permitted, provided the original author(s) or licensor are credited and that the original publication in this journal is cited, in accordance with accepted academic practice. No use, distribution or reproduction is permitted which does not comply with these terms. 